\title{
Genomic Expression in Peripheral Blood Mononuclear Cells (Pbmcs) of Patients with Hepatocellular Carcinoma (Hcc): Effect of Ablative Treatment
}

\section{Annamaria Gesualdo}

University of Bari: Universita degli Studi di Bari Aldo Moro

Francesca Passerini

University of Bari: Universita degli Studi di Bari Aldo Moro

\section{Simona D'Amore}

Cambridge University: University of Cambridge

Lucia Donatella Grimaldi

University of Bari: Universita degli Studi di Bari Aldo Moro

Antonino Noto

University of Bari: Universita degli Studi di Bari Aldo Moro

\section{Daniela Santovito}

University of Bari: Universita degli Studi di Bari Aldo Moro

\section{Giusi Graziano}

Center for Outcomes Research and Clinical Epidemiology

\section{Michele Milella}

University of Bari: Universita degli Studi di Bari Aldo Moro

\section{Giuseppe Marano}

University of Bari: Universita degli Studi di Bari Aldo Moro

Filomena Puntillo

University of Bari: Universita degli Studi di Bari Aldo Moro

Alessandra Mangia

IRCCS Ospedale Casa Sollievo della Sofferenza: Ospedale Casa Sollievo della Sofferenza

Vincenzo Ostilio Palmieri ( $\square$ vincenzoostilio.palmieri@uniba.it )

University of Bari: Universita degli Studi di Bari Aldo Moro https://orcid.org/0000-0002-7649-6028

\section{Research Article}

Keywords: Hepatocellular carcinoma, genomic expression, PBMCs, ablative treatment

Posted Date: August 13th, 2021 
DOl: https://doi.org/10.21203/rs.3.rs-765972/v1

License: (c) (1) This work is licensed under a Creative Commons Attribution 4.0 International License. Read Full License 


\section{Abstract}

Genome-wide association studies revealed potential driver genes involved in hepatocellular carcinoma (HCC) development thus providing putative therapeutic targets and predictive biomarkers for personalized therapy.

In the present study, we analyzed changes in the gene expression profile of circulating peripheral mononuclear cells (PBMCs) of HCC patients undergoing treatment in order to identify the gene signatures useful as biomarkers for prognostic classification. Patients (24) diagnosed with HCC and staged according to BCLC criteria were enrolled. All patients underwent treatment by radiofrequency ablation or chemoembolization and the effect of treatment was evaluated three months later. Patients were stratified into 2 subgroups: 11 with complete response; 13 with partial response. Furthermore, we added six patients (with stable or progressive disease) to the initial cohort. Microarray expression profiling on PBMCs isolated before and three months after the therapeutic procedure was performed in order to identify upregulated and downregulated genesin each patient. In addition, quantitative real-time PCR was used to confirm differentially expressed genes on microarray analysis. The most intriguing results were the upregulation at baseline of ENTPD1 (ectonucleoside triphosphate diphosphohydrolase-1, able to suppress the immune system response to malignancies) and the down-regulation of ICOS (inducible costimulator, involved in anti-tumour T cell response) in patients with partial response to treatment. To the best of our knowledge, there are no study relating ENTPD1 and ICOS gene signatures to outcome of treatment in HCC. Our findings highlight a new role for ENTPD1 and ICOS as useful biomarkers for prognostic classification of patients with liver cancer.

\section{Introduction}

Hepatocellular carcinoma (HCC) is the most frequent among primary malignancies of the liver and represents the third leading cause of cancer death worldwide, accounting for more than 600,000 deaths annually [1]. Despite being the leading cause of death in patients with compensated cirrhosis, $\mathrm{HCC}$ is typically diagnosed late in its course [2]. In this view, it is essential to recognize the major risk factors of $\mathrm{HCC}$, perform an accurate early diagnosis of cancer and choose the optimal treatment option. The most important risk factor for HCC are cirrhosis HCV-, HBV- or ethanol-related. Recently also NASH (NonAlcoholic Steato-Hepatitis) has been regarded as an emerging risk factor for cirrhosis and HCC in Western countries [3]. Staging and therapy of HCC is currently based on the Barcelona Clinic Liver Cancer (BCLC) classification that considers the number and size of lesions, performance status, Child-Pugh classification and portal hypertension $[4,5]$

A number of genome-wide studies exploring the liver cancer genome have become available in recent years providing gene sets to classify and predict clinical outcomes and helping to elucidate molecular pathogenesis and genetic heterogeneity of HCC [6]. Like other cancers, several subclasses of HCC have been identified with distinct characteristics, such as tumor size, rate of cancer cell proliferation, and vascular invasion, and ultimately different prognoses, which are associated to aberrant intracellular 
signal transduction pathways $[7,8,9]$. However, these preliminary studies on the mutational spectrum of HCC have focused on a limited number of candidate genes. Advances in genome-sequencing technologies have enabled simultaneous analysis of thousands of expressed genes, accelerating the search for additional novel and recurrently mutated genes [10]. In most of the study on the genomic profiling of HCC, transcriptomic changes have been identified in neoplastic tissue obtained from surgical specimens and matched with those present in surrounding normal hepatic tissue [11]. This procedure implies that only patients with indication to surgical treatment of $\mathrm{HCC}$ have been enrolled. Any genomic classification of HCC, therefore, may be applied only on HCC patients in whom a sample of neoplastic tissue and of surrounding tissue may be obtained. Over the past years the analysis of the gene expression profiling of purified RNA derived from peripheral blood mononuclear cells (PBMCs) has been proposed for identifying the gene signature of neoplastic tissue $[12,13]$ otherwise inaccessible or difficult to obtain [14]. Furthermore, PBMCs have been proposed for these purposes since their gene expression profiles are relatively constant and repeatable in different individuals [15]. Based on these considerations, a possible contribution to the identification of genes involved in the progression of HCC may be obtained from the analysis of genetic landscape of PBMC of patients with HCC stratified according to treatment outcome.

In the present study, we have analyzed the gene expression profiling of PBMCs of patients with HCC at baseline and three months after ablative treatment to deepen the current understanding of molecular pathways promoting HCC progression and to determine whether a disease signature may predict different responses to treatment of this complex and heterogeneous disease.

\section{Patients And Methods}

\section{Study Population and design of the study}

The study was conducted prospectively on 24 patients with diagnosis of HCC consecutively enrolled at the Clinica Medica "A. Murri" (Department of Biomedical Sciences and Human Oncology - Policlinico of Bari, Italy), in the period between January 2015 and January 2018. For the assessment of the quantitative real-time polymerase chain reaction (RTqPCR), we added six patients to the initial cohort.

The genomics experiments were conducted in collaboration with the Interdisciplinary Department of Medicine of University of Bari.

Characteristics of the study population are provided in Table 1. 
Table 1

Main clinical features of HCC patients included in the study

Total number of patients

Age (mean $\pm \mathrm{sd})$

Male/Female ( $n, \%)$

Cirrhosis etiology $(n, \%)$

$\mathrm{HCV}$

HBV

CHILD PUGH CLASS $(n, \%)$

CHILD A (5-6)

CHILD B (7-9)

$\operatorname{BCLC}$ STAGE $(n, \%)$

BCLC stadio A

BCLC stadio $B$

TREATMENT $(n, \%)$

RFA

TACE
30

$68.5 \pm 8.1$

$25(83.4 \%) 5(16.6 \%)$

$25(83.4 \%)$

$5(16.6 \%)$

$15(50 \%)$

$15(50 \%)$

$23(76.7 \%)$

$7(23.3 \%)$

$14(46.7 \%)$

BCLC: Barcelona Clinic Liver Cancer; RFA: RadioFrequency Ablation; TACE: TransArterial ChemoEmbolization

Diagnosis of HCC was based on the criteria of the International Working Party [16] and confirmed in all patients by the execution of diagnostic procedures (Computed Tomography-CT, Magnetic Resonance Imaging-MRI) and, if necessary, by liver biopsy and subsequent histological and cytological evaluation, as already described elsewhere [17].

UltraSound-guided biopsy was performed using an 18gauge needle-biopsy (Zanotti Medical Research, Suzzara, Italy). Specimens were routinely processed and were stained first with hematoxylin and eosin and then with immunohistochemistry.

For each patient, anthropometric (weight, height, body mass index, abdominal circumference) and clinical data (blood pressure and heart rate), biochemical parameters (complete blood count, liver function tests, coagulation tests, renal function, protein and electrolytes, lipids, alpha-fetoprotein, ammonia) and blood samples for genomic analysis before treatment (T0) and three months after the procedure ( $\mathrm{T} 1)$ were collected (Table 2). 
Table 2

Comparison of clinical parameters before (T0) and three months after treatment (T1) in all patients enrolled in the study (mean $\pm \mathrm{SD}$ )

\begin{tabular}{|c|c|c|c|}
\hline Clinical parameters & $\mathrm{TO}(\mathrm{M} \pm \mathrm{SD}) \mathrm{n}=30$ & $T 1(M \pm S D) n=30$ & $p$ \\
\hline $\mathrm{BMI}\left(\mathrm{kg} / \mathrm{m}^{2}\right)$ & $26.1 \pm 4.2$ & $26.3 \pm 4.3$ & n.s. \\
\hline Waist Circumference (cm) & $100.4 \pm 9.3$ & $101.3 \pm 10.6$ & n.s. \\
\hline Total cholesterol (mg/dl) & $142.1 \pm 43.1$ & $141.5 \pm 39.6$ & n.s. \\
\hline LDL-c (mg/dl) & $79.8 \pm 32.7$ & $81.1 \pm 25.4$ & n.s. \\
\hline $\mathrm{HDL}-\mathrm{c}(\mathrm{mg} / \mathrm{dl})$ & $44 \pm 15.7$ & $43.7 \pm 13.1$ & n.s. \\
\hline $\mathrm{TG}(\mathrm{mg} / \mathrm{dl})$ & $113.8 \pm 75.8$ & $113.3 \pm 63.4$ & n.s. \\
\hline Glucose (mg/dl) & $108.8 \pm 31.3$ & $103.2 \pm 28.2$ & 0.03 \\
\hline $\operatorname{CRP}(\mathrm{mg} / \mathrm{dl})$ & $6.8 \pm 5$ & $8.9 \pm 18.8$ & n.s. \\
\hline $\operatorname{ESR}(\mathrm{mm} / \mathrm{h})$ & $26.7 \pm 21.6$ & $28.3 \pm 28.4$ & n.s. \\
\hline AST (U/L) & $84 \pm 64.5$ & $79.6 \pm 66.5$ & n.s. \\
\hline $\operatorname{ALT}(\mathrm{U} / \mathrm{L})$ & $83 \pm 57$ & $65.4 \pm 56.5$ & 0.02 \\
\hline GGT (U/L) & $98.7 \pm 74$ & $92.7 \pm 72.9$ & n.s. \\
\hline $\operatorname{ALP}(\mathrm{U} / \mathrm{L})$ & $128.2 \pm 54.5$ & $148.1 \pm 96.9$ & n.s. \\
\hline Total bilirubin (mg/dl) & $1.2 \pm 0.7$ & $1.3 \pm 1$ & n.s. \\
\hline $\operatorname{AFP}(\mathrm{ng} / \mathrm{ml})$ & $219.5 \pm 628.8$ & $87.4 \pm 270.7$ & n.s. \\
\hline LDH & $205.4 \pm 54.3$ & $210.4 \pm 44.1$ & n.s. \\
\hline $\mathrm{HB}(\mathrm{g} / \mathrm{dl})$ & $13.1 \pm 1.7$ & $12.7 \pm 1.9$ & 0.01 \\
\hline WBC $\left(\times 10^{3} / \mathrm{u} / \mathrm{L}\right)$ & $5.2 \pm 2.1$ & $5.2 \pm 1.9$ & n.s. \\
\hline Neutrophils $\left(x 10^{3} / \mathrm{u} / \mathrm{L}\right)$ & $2.95 \pm 1.3$ & $3.1 \pm 1.8$ & n.s. \\
\hline Lymphocytes (x103/u/L) & $1.6 \pm 0.8$ & $1.5 \pm 0.7$ & n.s. \\
\hline N/L Neutrophils/Lymphocytes ratio & $2.1 \pm 1$ & $2.9 \pm 3.6$ & n.s. \\
\hline Platelets $\left(\times 10^{3} / \mathrm{u} / \mathrm{L}\right)$ & $131.4 \pm 85.8$ & $118.5 \pm 81.3$ & 0.02 \\
\hline
\end{tabular}

BMI: body mass index; LDL-C: low density lipoprotein cholesterol; HDL-C: high density lipoprotein cholesterol; TG: triglycerides; CRP: C-reactive protein; ESR: erythrocyte sedimentation rate; AST: aspartate aminotransferase; ALT: alanine aminotransferase; GGT: -glutamyltranspeptidase; ALP: alkaline phosphatase; AFP: AlphaFetoProtein; LDH: lactate dehydrogenase; HB: Haemoglobin; WBC: white blood cells. BUN: blood urea nitrogen; PT-INR pro-thrombin international normalised ratio. 


\begin{tabular}{|c|c|c|c|}
\hline Clinical parameters & $\mathrm{TO}(\mathrm{M} \pm \mathrm{SD}) \mathrm{n}=30$ & $\mathrm{~T} 1(\mathrm{M} \pm \mathrm{SD}) \mathrm{n}=\mathbf{3 0}$ & $\mathbf{p}$ \\
\hline Creatinine (mg/dl) & $0.9 \pm 0.3$ & $0.9 \pm 0.3$ & n.s. \\
\hline BUN Blood Urea Nitrogen (mg/dl) & $41.8 \pm 16.3$ & $44 \pm 21$ & n.s. \\
\hline Total serum proteins $(\mathrm{g} / \mathrm{dl})$ & $7.4 \pm 0.5$ & $7.01 \pm 0.7$ & 0.001 \\
\hline Albumin $(\mathrm{g} / \mathrm{dl})$ & $3.4 \pm 0.5$ & $3.4 \pm 0.7$ & n.s. \\
\hline Fibrinogen $(\mathrm{mg} / \mathrm{dl})$ & $232.2 \pm 47.3$ & $238.1 \pm 76.8$ & n.s. \\
\hline PT INR & $1.2 \pm 0.2$ & $1.2 \pm 0.2$ & n.s. \\
\hline Ammonia $\mu \mathrm{g} / \mathrm{dL}$ & $38.7 \pm 19.5$ & $47.9 \pm 23.2$ & 0.01 \\
\hline Total volume neoplasm (ml) & $40.7 \pm 88.6$ & $18.6 \pm 47.1$ & 0.0001 \\
\hline Child Pugh score & $6.1 \pm 1.1$ & $6.2 \pm 1.5$ & n.s. \\
\hline \multicolumn{4}{|c|}{$\begin{array}{l}\text { BMI: body mass index; LDL-C: Iow density lipoprotein cholesterol; HDL-C: high density lipoprotein } \\
\text { cholesterol; TG: triglycerides; CRP: C-reactive protein; ESR: erythrocyte sedimentation rate; AST: } \\
\text { aspartate aminotransferase; ALT: alanine aminotransferase; GGT: -glutamyltranspeptidase; ALP: } \\
\text { alkaline phosphatase; AFP: AlphaFetoProtein; LDH: lactate dehydrogenase; HB: Haemoglobin; WBC: } \\
\text { white blood cells. BUN: blood urea nitrogen; PT-INR pro-thrombin international normalised ratio. }\end{array}$} \\
\hline
\end{tabular}

To assess the effectiveness of treatment, each patient underwent CT or MRI three months after therapeutic procedures, according to the criteria for the evaluation of the response of solid tumors modified for hepatocellular carcinoma (mRECIST) [18].

The gene expression analysis was carried out based on the response to treatment: complete response (11 patients, CR), partial response (13 patients, PR). Patients added to the initial cohort for the RTqPCR validation were affected by stable and/or progression disease (6 patients, SD or PD).

Exclusion criteria were: HCC in stage BCLC D (in which only palliative treatment is indicated); indication to treatment with sorafenib; presence of other neoplastic disease; presence of clinically relevant renal, cardiac, cerebral or respiratory disease.

The study protocol was approved by the Ethical Committee of the Azienda Ospedaliero-Universitaria Policlinico di Bari, Italy.

Each procedure followed in the study was in accordance with the ethical standards of the responsible committee on human experimentation (institutional and national) and with the Helsinki Declaration of 1975, as revised in 2000 and 2008.

All patients signed an informed consent for the use of clinical data, and blood samples for scientific research purposes connected to this project.

\section{PBMCs isolation}


Blood samples were obtained for each patient at baseline and three months after therapeutic procedures. Fresh whole blood $(18 \mathrm{ml})$ was collected by standardized venipuncture in EDTA anti-coagulant tubes (Vacuette ${ }^{\circledR}$, Greiner Bio-One, Kremsmunster, Austria). Within $3 \mathrm{~h}$ of collection, blood was processed by density gradient centrifugation using Ficoll-PaqueTM PLUS (GE Healthcare, Orsay, France), according to a standard, previously validated protocol [19-21].

PBMCs isolated were stored at $-80^{\circ} \mathrm{C}$ until RNA extraction as described below.

\section{RNA extraction and quality control}

In order to perform the extraction of RNA, each aliquot of PBMCs was thawed, washed in $10 \mathrm{ml}$ PBS and centrifuged for $8 \mathrm{~min}$ at $1000 \mathrm{rcf}$ at $4^{\circ} \mathrm{C}$. RNA was extracted from the PBMCs pellet using QIAzolß Lysis Reagent (Qiagen, Hilden, Germany), according to the manufacturer's instructions. The RNA obtained was subjected to spectrophotometric analysis, to determine the concentration and purity (ratio OD260/OD280, pure if next to the value of 2), and to quality control, to assess the degree of degradation.

RNA integrity was assessed by Bio-Rad Experion ${ }^{\text {TM }}$ (Bio-Rad, Hercules, CA). The RNA quality was defined as a Relative Quality Index $(\mathrm{RQI})>7$, which is considered expression of excellent quality. All samples included in this study had a RQI above 8.

\section{Microarray analysis of PBMCs gene expression profiling}

Microarray gene expression analysis was conducted on RNA extracted from the PBMCs at baseline and three months after treatment. Whole RNA (400 ng) was used for cRNA synthesis using the Illumina Total Prep RNA Amplification kit (Ambion, Austin, TX, US) following the manufacturer's instructions, and randomly hybridized to the array. Whole-Genome gene expression experiments were conducted using the Illumina whole genome direct hybridization assay (HumanHT-12 v.4 Expression BeadChip Kit) on the Illumina microarray platform (Illumina iScan System).

Upon the manufacturer instructions, data were processed using the Illumina Genome Studio Software through specific algorithms of filtration and cleaning of the signal. All the items with detection $p$ value > 0.001 were excluded. Data were normalized together with the quantile method [22]. Background was subtracted. Final output consisted of normalized fluorescence intensity of each probe (AVG signal), representing the expression levels of each gene. We excluded genes discontinued or poorly annotated in NCBI Entrez Gene Database records. This initial screening allowed us to identify 4683 genes to be exported from Genome Studio Software on which, after transformation log2-transformed data, we thus performed the statistical analysis. The identification of genes which were up- or down-regulated in each group was performed by comparing gene expression in PBMCs at baseline and 3 months after treatment.

\section{Quantitative real-time polymerase chain reaction (RTqPCR)}

Gene expression of 17 genes, chosen by comparing the scientific literature for their role in carcinogenesis and particularly in HCC, was quantitatively assessed using Quantitative Real Time PCR (RT-qPCR). Samples were run in triplicate wells on each plate. cDNA was generated from $4 \mu \mathrm{g}$ total RNA using High 
Capacity DNA Archive Kit (Applied Biosystem, Foster City, CA) and following the manufacturer's instructions. mRNA expression levels were quantified using Power Sybr Green chemistry and normalized to cyclophilin mRNA levels. Relative quantification was performed using the $\Delta \Delta C T$ method. The values are expressed as mean \pm SEM.

TaqMan ${ }^{\circledR}$ and Gene ExpressionAssays and TaqMan ${ }^{\circledR}$ Universal PCR Master Mix with UNG following the instructions of the producer. The following conditions have been met: UNG incubation at $50^{\circ} \mathrm{C}$ for $2 \mathrm{~min}$ (only for TaqMan); then (for all experiments) denaturation at $95^{\circ} \mathrm{C}$ for $10 \mathrm{~min}$, followed by 40 cycles at $95^{\circ} \mathrm{C}$ for $15 \mathrm{~s}$, then at $60^{\circ} \mathrm{C}$ for $60 \mathrm{~s}$. The baseline values for amplification were set automatically and the threshold values were kept constant to obtain normalized cycle times and linear regression data. The individual PCR efficiency receptor was calculated from the slope of the resulting standard curves, using the formula $E=10-1$ / slope where $E$ is efficiency. The efficiency obtained was used to convert the cycle times from the logarithmic to the linear scale according to the formula E-ct. Normalized mRNA levels were expressed as arbitrary (relative) units and were obtained by dividing the corrected average for efficiency of the mRNA expression values of our genes for that of the cyclophilin mRNA of each sample. The values obtained were multiplied by 106 for the graphic representation of the arbitrary units (to avoid values below 1 when the expression of a gene is lower than that of the constitutive gene), and inserted as mean \pm SEM.

This dataset has been deposited in the National Center for Biotechnology Information Gene Expression Omnibus (GEO; http://ncbi.nim.nih.gov/geo) and is available quoting the GEO accession number GSE143004.

\section{Statistical analysis}

Data on clinical and bio-temporal variables are expressed as mean and standard deviations. The calculations on these variables were made with the NCSS 2007 software (Kaysville, UT, USA).

The differences between two groups were evaluated with Mann-Whitney U-test or Student's t test, when appropriate. The Pearson chi-square test was used to compare proportions. A two-tailed probability value $(P)$ of less than 0.05 was considered statistically significant.

All the 4683 genes identified were analyzed in a classical statistical setting. The evaluation of differences between the two groups of interest (partial response vs complete response) were assessed before (T0) and after (T1) treatment using the Mann-Whitney $U$ test. The test paired version (Wilcoxon signed-rank test) was also used to compare the pre-post mean values of partially responders and complete responders group, separately. Results are presented as mean \pm SEM. To overcome the multiple comparison problem and to control the error I type, the false discovery rate (fdr) with the BenjaminiHochberg method was calculated. Genes with a fold change $\geq 1.3$ and a P-values $<0.05$ were considered differentially expressed in statistical terms. All the analyses were performed with the R statistical software (version 3.1.2). 


\section{Results}

\section{Clinical and biochemical parameters}

Clinical characteristics and biochemical parameters of patients enrolled in the study are shown in Table 2 that summarizes the results obtained for each parameter, before (TO) and three months after treatment (T1).

At three months from the therapeutic procedure it was observed a decrease in blood glucose $(p=0.03)$, $\operatorname{ALT}(p=0.02)$, hemoglobin $(p=0.01)$, platelets $(p=0.02)$ and total serum proteins $(p=0.001)$ levels, total tumor volume $(p=0.0001)$ and an increase in ammonia $(p=0.01)$.

\section{Gene expression profiling in PBMCs after ablative treatment}

In order to study the effects induced by ablative treatment on PBMCs at transcriptional level, we performed gene expression microarrays on 11 patients with complete response to treatment and 13 subjects with partial response. At the paired analysis (T0, baseline values, vs. T1, after 3 months from the ablative procedure), we observed the modulation of 30 annotated genes ( 6 up-regulated and 24 downregulated; fold-change $\geq 1.3 ; p<0.05)$ in the PBMCs of patients with $C R$, and of 5 annotated genes ( 2 upregulated and 3 down-regulated; fold-change $\geq 1.3 ; p<0.05)$ in the PBMCs of patients with PR. The complete list of the genes differentially modulated is shown in Supplementary Table 1.

We then investigate differences in the PBMCs transcriptional profiles between patients with $\mathrm{CR}$ and subjects with PR; at baseline, 8 genes were differentially expressed ( 5 upregulated and 3 downregulated; fold-change $\geq 1.3 ; p<0.05$ ) between $C R$ and PR patients, while at 3 months 23 genes were differentially expressed between CR and PR groups ( 14 upregulated and 9 downregulated; fold-change $\geq 1.3 ; p<0.05$ ).

Table 3 shows the list of genes that have a statistically significant $(p<0.05)$ difference in the expression levels between the various groups. 
Table 3

List of genes that were statistically significant $(p<0.05)$ at microarray analysis (in underlined italics the genes on which RT-qPCR was performed)

\begin{tabular}{|c|c|}
\hline UPREGULATED - CR vs PR Time 0 & UPREGULATED - CR T0 vs T1 \\
\hline SNCA & DPYD \\
\hline PHF13 & EAF2 \\
\hline ENTPD1 & HSD17B4 \\
\hline C14orf2 & WASPIP \\
\hline NP & XRN2 \\
\hline DOWNREGULATED - CR vs PR Time 0 & DERA \\
\hline ICOS & DOWNREGULATED - CR T0 vs T1 \\
\hline TRIM4 & ZC3H12A \\
\hline LEPROTL 1 & RNU1-5 \\
\hline UPREGULATED CR vs PR - Time 1 & CUL9 \\
\hline RN5S9 & STAB1 \\
\hline GAA & RNU1G2 \\
\hline VPS18 & VPS18 \\
\hline SASH3 & CORO7 \\
\hline DNM2 & RNU1-3 \\
\hline DDT & LTB4R \\
\hline C9orf119 & SNORD3A \\
\hline ESRRA & SAPS1 \\
\hline ACIN1 & PREX1 \\
\hline CORO7 & DDX10 \\
\hline TRAPPC5 & RNU1A3 \\
\hline FHOD1 & ACTN4 \\
\hline MY01F & FAM195B \\
\hline PPP1R12C & CCDC88C \\
\hline DOWNREGULATED CR vs PR Time 1 & $\mathrm{H} 1 \mathrm{FX}$ \\
\hline C7orf28B & STAT5A \\
\hline
\end{tabular}


MS4A7

TRIM4

$\mathrm{ZCCHC7}$

MFF

DYNC112

$\mathrm{HSPH} 1$
ACIN1

ATXN7L3

CSRP1

FAM53B

UPREGULATED - PR T0 vs T1

APOBEC3A

LFNG

DOWNREGULATED - PR T0 vs T1

IDI1

KLF9

CR: Complete Response $(n=11)$; PR: Partial Response $(n=12)$; T0: before treatment; T1: three months after treatment. See text for more information on the denomination of genes

No difference in the levels of gene expression was observed in the group of stable/progressive disease before and after treatment at microarray analysis.

\section{Quantitative RT-PCR (qPCR) of changes in PBMCs gene expression before and after treatment}

To confirm the microarray analysis result, RTqPCR validation was performed to investigate RNA levels of 17 genes differently expressed (fold-change $\geq 1.3 ; p<0.05$ ) between the $P R$ and $C R$ patients, on the basis of their biological relevance in cancer evolution in some cases also of hepatic origin: CCDC88C (coiledcoil domain containing 88C), CORO7 (coronin-7), LTBR4 (Leukotriene B4 Receptor), KLF9 (Kruppel like factor 9), PREX1 (phosphatidylinositol-3,4,5-trisphosphate dependent Rac exchange factor 2), ACTN4 (alpha actinin 4), XRN2 (exoribonuclease 2), STAB1 (stabilin-1), STAT5 (Signal Transducers and Activators of Transcription 5A, ZC3H12A (zinc finger CCCH-type containing 12A), ENTPD1 (nucleoside triphosphate diphosphohydrolase-1), EAF2 (ELL-associated factor 2), HSD17B4 (17 $\beta$ hydroxysteroid dehydrogenase type 4), ACIN1 (apoptotic chromatin condensation inducer in the nucleus), CUL9 (cullin 9), SNORD3A (Small Nucleolar RNA, C/ D Box 3A), ICOS (inducible T cell co-stimulator).

Only part of the differential changes in the expression of the candidate genes observed at the microarray analysis was confirmedby RTqPCR in the expanded validation cohort.

The results of the RT-qPCR are illustrated considering the entire sample of patients $(n=30)$, patients with complete response $(C R)(n=11)$, those with partial response $(P R)(n=13)$ and those with stable or progression disease $(S D / P D)(n=6)$, both for the down-regulated and up-regulated genes. 
At the paired analysis (T0 vs. T1), we confirmed the suppression of KLF9 in patient with partial response to treatment $(0.96 \pm 0.07$ vs $0.74 \pm 0.04, p<0.04)$, while the down-regulation of CCDC88C, CORO7, LTBR4, KLF9 and PREX1 was confirmed only in patients with complete response to treatment (Table 4).

Table 4

Results of the amplification with RT-qPCR of the genes resulted downregulated at the microarray assay, at time 0 (T0) and after treatment (T1) in patients with complete $(n=11)$ and partial $(n=13)$ response to treatment

\begin{tabular}{|llll|}
\hline GENES & T0 & T1 & p \\
\hline CCDC88C & $1.07 \pm 0.04$ & $0.87 \pm 0.01$ & 0.04 \\
\hline COR07 & $0.99 \pm 0.01$ & $0.22 \pm 0.04$ & 0.00045 \\
\hline LTBR4 & $1.54 \pm 0.31$ & $0.35 \pm 0.13$ & 0.03 \\
\hline KLF9 & $1.27 \pm 0.21$ & $0.78 \pm 0.04$ & $0.03^{*}$ \\
\hline PREX1 & $1.11 \pm 0.06$ & $0.72 \pm 0.07$ & 0.04 \\
\hline ACTN4 & $1.06 \pm 0.15$ & $0.48 \pm 0.28$ & NS \\
\hline XRN2 & $0.58 \pm 0.19$ & $0.46 \pm 0.29$ & NS \\
\hline STAB1 & $1.14 \pm 0.10$ & $0.65 \pm 0.16$ & NS \\
\hline STAT5 & $0.82 \pm 0.06$ & $0.58 \pm 0.21$ & NS \\
\hline ZC3H12A & $1.28 \pm 0.16$ & $0.61 \pm 0.15$ & \\
\hline $\begin{array}{l}\text { Results are expressed as Mean }+ \text { SEM of relative mRNA. See text for more information on the } \\
\text { denomination of genes }\end{array}$ & & \\
\hline * For KLF9, the down regulation of expression is only confirmed in patients with partial response \\
\hline
\end{tabular}

In patients with complete or partial response, the only gene for which up regulation was confirmed was EAF2 (Table 5). 
Table 5

Results of the amplification with RT-qPCR of the genes resulted upregulated at the microarray assay, at time 0 (T0) and after treatment (T1) on patients with complete $(n=11)$ and partial $(n=13)$ response to treatment

\begin{tabular}{|llll|}
\hline GENES & T0 & T1 & P \\
\hline EAF2 & $0.91 \pm 0.10$ & $1.59 \pm 0.07$ & 0.015 \\
\hline HSD17B4 & $2.44 \pm 0.91$ & $3.98 \pm 1.16$ & NS \\
\hline ACIN1 & $1.12 \pm 0.11$ & $1.26 \pm 0.03$ & NS \\
\hline CUL9 & $1.25 \pm 0.13$ & $1.12 \pm 0.10$ & NS \\
\hline SNORD3A & $1.32 \pm 0.20$ & $1.10 \pm 0.03$ & NS \\
\hline $\begin{array}{l}\text { Results are expressed as Mean } \pm \text { SEM of relative mRNA. See text for more information on the } \\
\text { denomination of genes }\end{array}$
\end{tabular}

Finally, in patients with stable or progressive disease none of the genes considered resulted significantly modified after treatment.

The comparison between patients with complete response and those with partial response at time 0 revealed an overexpression of ENTPD1 and a suppression of ICOS in patients who then presented a partial response to treatment (Table 6 and Fig. 1).

Table 6

Results of the amplification with RT-qPCR of the genes resulted modified at the microarray assay, in the comparison at time $0(T 0)$ of patients with CR $(n=11)$ and of patients with PR $(n=13)$

\begin{tabular}{|llll|}
\hline GENES & CR & PR & P \\
\hline ENTPD1 & $0.91 \pm 0.08$ & $1.31 \pm 0.07$ & 0.01 \\
\hline ICOS & $2.11 \pm 0.55$ & $0.92 \pm 0.16$ & 0.03 \\
\hline $\begin{array}{l}\text { Results are expressed as Mean } \pm \text { SEM of relative mRNA. See text for more information on the } \\
\text { denomination of genes. CR: Complete Response; PR: Partial Response }\end{array}$ & \\
\hline
\end{tabular}

Results of the comparison T0 vs T1 for all genes in which at RT-qPCR gene expression modifications were statistically significant are further reported in Fig. 2.

\section{Discussion}

In the field of liver cancer research, a number of genetic and genome-wide profiling studies have detailed the genomic landscape of HCC and advanced the understanding of the underlying mechanisms involved in the multi-step hepatocarcinogenesis. Deregulated, non-resolving inflammation is thought to be central to the development and progression of HCC: emerging evidence has clarified that HCC-associated inflammation can be driven by genetic events promoting the secretion of factors guiding the build-up of 
an inflammatory microenvironment (intrinsic pathway) and by exogenous factors able to maintain a persistent inflammatory response (extrinsic pathway) (PMID 29872724). In this study, we have employed microarrays to profile the specific gene expression signature in circulating PBMCs of patients with HCC that could be associated with tumor progression and different response to treatment.

Results have been analyzed in relation to two main factors: the degree of response to treatment (complete or partial) and time of analysis of genes expression: before treatment (time 0 ) and three months after treatment (time 1).

From the analysis of the results, it emerged first that at time 0 there was no significant difference between the groups of subjects at RTqPCR level on circulating PBMCs, except for two genes: ENTPD1 and ICOS, respectively over-expressed and under-expressed at baseline in patients with partial response to ablative treatment.

The ENTPD1 gene (nucleoside triphosphate diphosphohydrolase-1) encodes an enzyme expressed on many cell types including leukocytes, endothelial cells, Kuppfer cells, regulatory T lymphocytes [23-25]. The enzyme catalyzes the degradation to AMP of extracellular ATP, which plays an anti-tumor role by activating the immune response against the tumor and directly in the death of neoplastic cells. Furthermore, the gene expression at the level of the regulatory $T$ lymphocytes inhibits the antitumor activity of natural killer cells and promotes tumor growth, while the expression at the endothelial cell level facilitates angiogenesis [26].

Over-expression of ENTPD1 has been demonstrated in several cancers including melanoma [27], leukemia [28], pancreatic adenocarcinoma [29], ovarian carcinoma [30], while in colorectal adenocarcinoma lower expression levels of gene have been correlated to a lower metastatic tumor power and a longer survival [31].

In a study on 324 patients underwent to surgical resection for HCC, Cai et al have demonstrated that the expression of the ENTPD1 gene at the level of tumor cells and normal hepatocyte cell lines is an independent predictor of worse prognosis after resection of the tumor [32]. According to the data of the literature, in our study the gene is significantly upregulated at T0 in patients who reached the partial response at three months from treatment, thus delineating a prognostically unfavorable gene expression profile in terms of response to treatment. This novel data seems to confirm the observation of Bastid et al [33] that CD39/ENTPD1 inhibition may have anticancer activity and that ENTPD1 inhibitory compounds have a possible favorable safety profile.

The ICOS gene (Inducible T Cell Costimulator), is a CD28 family receptor, which modulates costimulatory signals necessary to fully activate lymphocyte $T$ cells. It is expressed on $T$ cells and, interacting with its ICOSL ligand, present on APCs (antigen presenting cells, such as B cells, macrophages and dendritic cells) and on some tumor cells, it stimulates T lymphocyte activity. The role of ICOS in oncogenesis is twofold, because, depending on the context, it can stimulate the CD8 + T lymphocytes and their antitumor action or activate the T-regulators, favoring the immune escape [34]. 
The ICOS + T-Regs are the predominant component in the microenvironment of HCC, in fact they are much more present, both in the liver and in the plasma, of affected subjects, compared to controls and, repressing the immune response against the tumor, correlate with a worse prognosis [35].

Studies on HepG2 cells show that the ICOS-ICOSL pathway is fundamental for neoplastic progression (if silenced, cell proliferation and invasion are inhibited) and its action is mediated by the PI3K pathway [36]. Similar results have also been confirmed on other tumors (breast, stomach, ovary) [37-39]. In some tumors, such as the colon, there are opposite results because CD4 + ICOS + promote the action of Th1 which inhibit tumor invasion and stimulate the CD8 + response against neoplastic cells [40].

In an apparently contrasting way to these data, in our study ICOS was statistically significantly downregulated at T0 in patients who then reported RP to treatment. However, it has been shown that there is a different distribution of Treg lymphocytes, which are significantly more concentrated at the tumor level than in the peritumoral tissue and in the peripheral blood. Therefore our result, which deserves to be reconsidered on a larger sample of subjects, can be justified, at least in part, by the fact that this cellular population is more represented at the tumor level, thus constituting a smaller proportion of the cells that represent the PBMCs whose gene expression has been assessed [41].

Apart from the significant exceptions of ENTPD1 and ICOS genes, the gene expression at RTqPCR of patients' PBMCs is not modified at time 0 and therefore we can assume that any difference in gene expression is due exclusively to the effect of the treatment that is the local consequences on the tumor microenvironment of the ablation of the tumor.

To the best of our knowledge, there is no study in literature in which the effect of treatment of HCC on the genomic expression in circulating PBMCs was evaluated.

A first consideration is that the treatment of the tumor can determine significant changes in peripheral gene expression. This result is even more surprising considering that it has also been detected in patients in whom the volume of the neoplasm is relatively small (average volume of $11.6 \mathrm{ml}$ in patients who have obtained a complete response).

We have documented variation of expression subsequent to treatment of six genes.

The CCDC88C gene, coding for the Daple protein, which belongs to the family of non-receptor guanine nucleotide exchange factors (GEF), acts on G1a by increasing the non-canonical way of Wnt, implicated in the modulation of cell motility [42]. It acts as an oncosuppressor in normal epithelial cells, while it is upregulated in tumor cells, which acquire particular invasive capacity. Gene expression levels have been shown to be increased in colorectal cancer and, to a greater extent, in hepatic and pulmonary metastases, emphasizing the role of GEF in tumor progression [43].

In our study the gene is downregulated at $\mathrm{T} 1$ in patients with complete response to treatment, confirming the fact that tumor ablation is accompanied by the down regulation of pathways that contribute to carcinogenesis, such as that of GEF family. 
The CORO 7 gene codes for coronin 7 protein, which belongs to the family of coronins, membrane proteins that have different roles in maintenance of cellular structure, in cell-cell and matrix-cell interactions, in cytoskeletal organization, in chemotaxis and in cellular migration. The composition and characteristics of cell membrane proteins, including coronins, undergo rearrangement during malignant transformation. For these reasons these proteins can be considered tumor biomarkers. Wu et al. analyzed the expression profile of coronin-1C in two HCC cell lines in mouse: a higher level of protein expression correlates with a more invasive neoplastic phenotype and with a higher frequency of pulmonary metastases. In humans, immunohistochemical studies of biopsy samples of HCC have shown that patients with higher expression levels of coronin-1 exhibited a more advanced state of disease [44]. Wang et al. demonstrated the overexpression of coronin-1C protein in HCC samples in comparison to the surrounding healthy parenchyma; moreover expression levels correlates with the proliferation and metastatic potential of HCC cells, through activation of Rac-1 [45]. Gao et al. have shown overexpression of coronin 3 in hepatocellular cells [46]. In our study, confirming the data reported in the literature, gene expression levels are downregulated in patients with complete response to treatment, while they do not vary in patients with stable disease.

The LTB4R gene codes for the Leukotriene B4 receptor, a pro-inflammatory eicosanoid derived from arachidonic acid, which acts as a chemoattractant for phagocytes. It is precociously up-regulated in many tumors (ovary, breast, liver, esophagus, colon), where it acts as an oncogene through various mechanisms: for example, it negatively regulates the anti-proliferative action of TGF- $\beta 1$ in breast carcinoma [47] and mediates proliferation induced by the oncogenic protein $X$ of hepatitis $B$ virus (HBX) in HBV-mediated HCC [48]. Because its upregulation correlates with a worse outcome, it has been shown to be a valid prognostic marker and a possible therapeutic target. The combined use of Celecoxib (COX2inhibitor) and MK886 (5-lipoxygenase inhibitor), in pancreatic and colorectal carcinomas, inhibits tumor growth through various mechanisms, including the suppression of overexpression of LTB4R by MK886 [49]. As expected on the data of the literature, in our study the gene is downregulated in patients who have achieved complete response to treatment, while it does not vary in patients with stable disease.

The KLF9 gene encodes for a factor belonging to the KLF (Kruppel-like factors) family, involved in cell proliferation and differentiation, both in physiological conditions and malignant transformation, through the link with the promoter sequences of target genes that can be activated or suppressed [50]. Fu DZ and coll. reported that KLF9 mRNA and protein levels were decreased in HCC tissues compared with normal liver tissues and the upregulation of KLF9 expression has antiproliferation and pro-apoptosis properties in HepG2 cells [51]. Sun $J$ et al. found that by binding to the p53 promoter, KLF9 upregulates p53 levels and KLF9 overexpression significantly promotes tumor regression in the xenograft model [52]. These studies demonstrate the oncosuppressive role of KLF9 in HCC.

PREX1, phosphatidylinositol-3,4,5-trisphosphate dependent Rac exchange factor 2, encodes for a protein belonging to the RacGEFs family (Rac guanine nucleotide exchange factors) and is regulated with a positive feedback mechanism, by PI3K. RacGEFs promote Rac activation, involved in cytoskeletal rearrangement, cell migration and adhesion, and ROS production [53]. P-Rex1 acts as a oncogene and its 
mRNA is upregulated in breast, thyroid, prostate, lymphoid, ovarian, kidney, adrenal and melanoma tumors. At the protein level, P-Rex 1 overexpression has only been confirmed in breast cancer, prostate cancer, and melanoma [54-56]. High P-Rex1 protein expression has been linked to decreased diseasefree breast cancer patient survival [54].

In our study the gene is significantly down regulated in patients who have achieved complete response to treatment, thus emphasizing its possible role in hepatocarcinogenesis, even if any clear evidence is still present in the literature.

EAF2, ELL-associated factor 2, interacts with RNA polymerase II and promotes the transcription elongation phase. It is a tumor suppressor gene and its expression is downregulated in human prostate cancer specimens and cell lines [57]. Furthermore, EAF2 knockout mice developed lung adenocarcinoma, hepatocellular carcinoma, B cell lymphoma and high-grade murine prostatic intraepithelial neoplasia (mPIN) [58].

The altered pathways due to its down-regulation are multiple: it is implicated in the non-homologous mechanism of DNA repair (NHEJ); interacts with p53, synergizing its oncosuppressive effects; antagonizes the effects of the WNT / beta-catenin pathway, constitutively activated in many tumors. Furthermore, in knock-out mice for EAF2 there is a deficiency of the anti-angiogenic trombospondin 1 protein and a consequent increase in hepatic angiogenesis [59-61].

In our study, the gene is upregulated $(p<0.05)$ in patients who have achieved complete response to treatment. This result is apparently in contrast to the few data in the literature on the relationship between its expression and the development of hepatocellular carcinoma and deserves further studies to understand if the pathways involved in its upregulation after tumor ablation coincide with those detected until now only on an experimental basis.

In conclusion, the analysis of the genomic profile of circulating PBMCs in patients with hepatocellular carcinoma treated with locoregional procedure showed significant variation in the expression of some genes.

The amplification by RT-qPCR confirmed the data obtained from the analysis of microarrays on the different expression of some genes both in the comparison of patients with $\mathrm{CR}$ and PR before treatment, and in the comparison between $\mathrm{T} 0$ and $\mathrm{T} 1$ in patients who reported complete response to therapy.

The ablation of hepatocellular nodules with radiofrequency or chemoembolization can determine a significant modification of gene expression in circulating PBMCs, which is therefore conditioned by the extent of tumor necrosis and by the characteristics of the tumor microenvironment.

The most intriguing results were the upregulation at baseline of ENTPD1 and the down-regulation of ICOS in patients with partial response to treatment. To the best of our knowledge, there are no study relating ENTPD1 and ICOS gene signatures to outcome of treatment in HCC patients. Our findings highlight a new 
role for ENTPD1 and ICOS as useful biomarkers for prognostic classification and therapeutic stratification of patients with liver cancer.

This assumes an important biological and prognostic value in order to better understand the pathogenetic mechanisms underlying the carcinogenesis and in the perspective of the development of a target therapy for patients affected by this tumor.

\section{Declarations}

\section{Acknowledgments}

The authors thank Marica Cariello and Antonio Moschetta (Interdisciplinary Department of Medicine of University of Bari, Italy) for their outstanding contribution to the project administration and supervision.

Funding: PRIN 2017, Minister of University, Protocol 2017EKMFTN_004

Conflicts of interest/Competing interests: no conflict of interest or competing interest

Availability of data and material: any supplementary data on this research is available

Code availability: any data on the software employed for statistical analysis are available

Authors' contributions:

Conceptualization: Vincenzo O. Palmieri

Formal analysis: Michele Milella, Giuseppe Marano, Filomena Puntillo, Alessandra Mangia

Project administration: Annamaria Gesualdo, Francesca Passerini, Daniela Santovito, Antonino Noto, Vincenzo O. Palmieri

Supervision: Alessandra Mangia

Validation: Annamaria Gesualdo, Francesca Passerini, Vincenzo O. Palmieri

Writing - original draft: Annamaria Gesualdo, Francesca Passerini, Antonino Noto, Vincenzo O. Palmieri

Writing - review \& editing: Annamaria Gesualdo, Francesca Passerini, Lucia Donatella Grimaldi, Vincenzo O. Palmieri, Simona D’Amore, Giusi Graziano

Ethics approval: The study protocol was approved by the Ethical Committee of the Azienda OspedalieroUniversitaria Policlinico di Bari, Italy.

Each procedure followed in the study was in accordance with the ethical standards of the responsible committee on human experimentation (institutional and national) and with the Helsinki Declaration of 1975, as revised in 2000 and 2008. 
All patients signed an informed consent for the use of clinical data, and blood samples for scientific research purposes connected to this project.

\section{References}

1. Forner A, Llovet JM, Bruix J. Hepatocellular carcinoma. Lancet 2012,379:1245-1255.

2. Ferlay J, Shin HR, Bray F, Forman D, Mathers C, Parkin DM. Estimates of worldwide burden of cancer in 2008: GLOBOCAN 2008. Int J Cancer 2010, 127: 2893-2917.

3. El-Serag HB. Epidemiology of Viral Hepatitis and Hepatocellular Carcinoma. Gastroenterology 2012, 142: 1264-1273.

4. European Association For The Study Of The Liver. EASL Clinical Practice Guidelines: Management of hepatocellular carcinoma. J Hepatol 2018, 69: 182-236

5. Marrero JA, Kulik LM, Sirlin CB et al. Diagnosis, Staging, and Management of Hepatocellular Carcinoma: 2018 Practice Guidance by the American Association for the Study of Liver Diseases Hepatology 2018, 68: 723-750

6. Chen X, Cheung ST, So S et al. Gene expression patterns in human liver cancers. Mol Biol Cell 2002,13:1929-1939.

7. Hoshida Y, Nijman SM, Kobayashi M et al. Integrative transcriptome analysis reveals common molecular subclasses of human hepatocellular carcinoma. Cancer Res 2009,69:7385-7392.

8. Nault JC, Zucman-Rossi J. Genetics of hepatobiliary carcinogenesis. Semin Liver Dis 2011,31:173187.

9. Zender L, Villanueva A, Tovar V, Sia D, Chiang DY, Llovet JM. Cancer gene discovery in hepatocellular carcinoma. J Hepatol 2010,52: 921-929.

10. Cleary SP, Jeck WR, Zhao $X$ et al. Identification of driver genes in hepatocellular carcinoma by exome sequencing. Hepatology. 2013,58:1693-1702.

11. Lamb JR, Zhang C, Xie T et al. Predictive genes in adjacent normal tissue are preferentially altered by sCNV during tumorigenesis in liver cancer and may rate limiting. PLoS One. 2011,6(7):e20090. DOI: 10.1371/journal.pone.0020090

12. Shi M, Chen MS, Sekar K, Tan CK, Ooi LL, Hui KM. A blood based three-gene signature for the noninvasive detection of early human hepatocellular carcinoma. Eur J Cancer. 2014,50:928-936.

13. Zhao L, Mou DC, Peng JR, Huang L, Wu ZA, Leng XS. Diagnostic value of cancer-testis antigen mRNA in peripheral blood from hepatocellular carcinoma patients. World J Gastroenterol. 2010 Aug 28,16(32):4072-4078.

14. Whitney AR, Diehn M, Popper SJ et al. Individuality and variation in gene expression patterns in human blood. Proc Natl Acad Sci U S A. 2003,100:1896-1901.

15. Eady JJ, Wortley GM, Wormstone YM et al. Variation in gene expression profiles of peripheral blood mononuclear cells from healthy volunteers. Physiol Genomics 2005,22:402-411. 
16. International Working Party. Terminology of nodular hepatocellular lesions. Hepatology 1995, 22: 983-993

17. Palmieri VO, Santovito D, Margari $F$ et al. Psychopathological profile and health-related quality of life (HRQOL) in patients with hepatocellular carcinoma (HCC) in cirrosis. Clin Exp Med. 2013 Dec 10. DOI:10.1007/s10238-013-0267-0.

18. Lencioni R, Llovet JM. Modified RECIST (mRECIST) assessment for hepatocellular carcinoma. Semin Liver Dis 2010, 30:52-60.

19. Boyum A. Isolation of leucocytes from human blood. A two-phase system for re- moval of red cells with methylcellulose as erythrocyte-aggregating agent. Scand. J. Clin. Lab. Invest. 1968, 97: 9-29.

20. Cariello M, Simone S, Loverre A et al. Coagulation activation is associated with nicotinamide adenine dinucleotide phosphate oxidase-dependent reactive oxygen species generation in hemodialysis patients. Antioxid. Redox Signal. 2012, 16: 428-439.

21. D'Amore S, Vacca M, Graziano $G$ et al. Nuclear receptors expression chart in peripheral blood mononuclear cells identifies patients with Metabolic Syndrome. Biochimica et Biophysica Acta 2013, 12: $2289-2301$

22. Rao YL, Lee $Y$, Jarjoura $D$ et al. A comparison of normalization techniques for microRNA microarray data. Stat Appl Genet Mol Biol. 2008,7(1): Article22. doi: 10.2202/1544-6115.1287.

23. Kas-Deelen AM, Bakker WW, Olinga $P$ et al. Cytomegalovirus infection increases the expression and activity of ecto-ATPase (CD39) and ecto- 5'nucleotidase (CD73) on endothelial cells. FEBS Lett 2001,491:21-25.

24. Pulte ED, Broekman MJ, Olson KE et al. CD39/NTPDase-1 activity and expression in normal leukocytes. Thromb Res 2007,121:309-317

25. Dranoff JA, Ogawa M, Kruglov EA et al. Expression of P2Y nucleotide receptors and ectonucleotidases in quiescent and activated rat hepatic stellate cells. Am J Physiol Gastrointest Liver Physiol 2004,287:G417-G424

26. Feng L, Sun X, Csizmadia E et al. Vascular CD39/ENTPD1 directly promotes tumor cell growth by scavenging extracellular adenosine triphosphate. Neoplasia 2011,13:206-216.

27. Dzhandzhugazyan KN, Kirkin AF, Straten Pt, Zeuthen J. Ecto-ATP diphosphohydrolase/CD39 is overexpressed in differentiated human melanomas. FEBS Lett 1998, 430:227-230.

28. Pulte D, Furman RR, Broekman MJ et al. CD39 expression on T lymphocytes correlates with severity of disease in patients with chronic lymphocytic leukemia. Clin Lymphoma Myeloma Leuk 2011,11: 367-367.

29. Künzli BM, Berberat PO, Giese $T$ et al. Upregulation of CD39/NTPDases and P2 receptors in human pancreatic disease. Am J Physiol Gastrointest Liver Physiol 2007, 292: G223-G230.

30. Häusler SFM, Montalbán del Barrio I, Strohschein J et al. Ectonucleotidases CD39 and CD73 on OvCA cells are potent adenosine generating enzymes responsible for adenosine receptor 2Adependent suppression of T cell function and NK cell cytotoxicity. Cancer Immunol Immunother 2011, 60:1405-1418. 
31. Künzli BM, Bernlochner MI, Rath S et al. Impact of CD39 and purinergic signaling on the growth and metastasis of colorectal cancer. Purinergic Signal 2011, 7:231-241.

32. Cai XY, Ni XC, Yi Y et al. Overexpression of CD39 in hepatocellular carcinoma is an independent indicator of poor outcome after radical resection. Medicine (Baltimore). 2016 Oct,95(40):e4989. DOI: 10.1097/MD.0000000000004989.

33. Bastid J, Cottalorda-Regairaz A, Alberici G, Bonnefoy N, Eliaou JF, Bensussan A. ENTPD1/CD39 is a promising therapeutic target in oncology. Oncogene. 2013, 32:1743-51. doi: 10.1038/onc.2012.269. Epub 2012 Jul 2.

34. Amatore F, Gorvel L, Olive D. Inducible Co-Stimulator (ICOS) as a potential therapeutic target for anticancer therapy. Expert Opin Ther Targets. 2018, 22:343-351.

35. Tu JF, Ding YH, Ying XH et al. Regulatory T cells, especially ICOS ${ }^{+} \mathrm{FOXP3}^{+}$regulatory $\mathrm{T}$ cells, are increased in the hepatocellular carcinoma microenvironment and predict reduced survival. Sci Rep. 2016, 6:35056. DOI: 10.1038/srep35056.

36. Wei Y, Wang Y, Zang A et al. Inducible T-cell co-stimulators regulate the proliferation and invasion of human hepatocellular carcinoma HepG2 cells. Biol Res. 2018, 51: 2. DOI: 10.1186/s40659-017-01507.

37. Conrad C, Gregorio J, Wang YH et al. Plasmacytoid dendritic cells promote immunosuppression in ovarian cancer via ICOS costimulation of Foxp3(+) T-regulatory cells. Cancer Res. 2012, 72: 52405249. DOI: 10.1158/0008-5472.CAN-12-2271.

38. Faget J, Sisirak V, Blay JY, Caux C, Bendriss-Vermare N, Ménétrier-Caux C. ICOS is associated with poor prognosis in breast cancer as it promotes the amplification of immunosuppressive CD4 ${ }^{+} \mathrm{T}$ cells by plasmacytoid dendritic cells. Oncoimmunology. 2013, 2: e23185. DOI: 10.4161/onci.23185.

39. Huang XM, Liu XS, Lin XK et al. Role of plasmacytoid dendritic cells and inducible costimulatorpositive regulatory $\mathrm{T}$ cells in the immunosuppression microenvironment of gastric cancer. Cancer Sci. 2014 105: 150-158. DOI: 10.1111/cas.12327.

40. Zhang Y, Luo Y, Qin SL et al. The clinical impact of ICOS signal in colorectal cancer patients. Oncoimmunology. 2016, 5:e1141857. DOI:10.1080/ 2162402X. 2016.1141857.

41. Li W, Han J, Wu H. Regulatory T-cells promote hepatitis B virus infection and hepatocellular carcinoma progression. Chronic Dis Transl Med. 2016, 2: 67-80.

42. Ara $\mathrm{H}$, Takagishi $\mathrm{M}$, Enomoto A et al. Role for Daple in non-canonical Wnt signaling during gastric cancer invasion and metastasis. Cancer Sci. 2016, 107:133-139. DOI: 10.1111/cas.12848.

43. Barbazan J, Dunkel Y, Li H et al. Prognostic impact of modulators of G proteins in circulating tumor cells from patients with metastatic colorectal cancer. Sci Rep. 2016 Feb 26,6:22112. DOI: $10.1038 / \operatorname{srep} 22112$.

44. Wu L, Peng CW, Hou JX et al. Coronin-1C is a novel biomarker for hepatocellular carcinoma invasive progression identified by proteomics analysis and clinical validation. J Exp Clin Cancer Res. 2010, 24, 29:17. DOI: 10.1186/1756-9966-29-17. 
45. Wang ZG, Jia MK, Cao H, Bian P, Fang XD. Knockdown of Coronin-1C disrupts Rac1 activation and impairs tumorigenic potential in hepatocellular carcinoma cells. Oncol Rep. 2013, 29:1066-1072. DOI: 10.3892/or.2012.2216.

46. Gao Y, Li L, Xing X et al. Coronin 3 negatively regulates G6PC3 in HepG2 cells, as identified by label-free mass-spectrometry. Mol Med Rep. 2017, 16: 3407-3414. DOI: 10.3892/mmr.2017.7002.

47. Jeon WK, Choi J, Park SJ et al. The proinflammatory LTB4/BLT1 signal axis confers resistance to TGF- 31 -induced growth inhibition by targeting Smad3 linker region. Oncotarget 2015, 6: 4165041666.

48. Wang Q, Zhang W, Liu Q et al. A mutant of hepatitis B virus X protein (HBxDelta127) promotes cell growth through a positive feedback loop involving 5-lipoxygenase and fatty acid synthase. Neoplasia. 2010, 12: 103-115.

49. Ding X, Zhu C, Qiang H, Zhou X, Zhou G. Enhancing antitumor effects in pancreatic cancer cells by combined use of COX-2 and 5-LOX inhibitors. Biomed Pharmacother. 2011, 65: 486-490.

50. Bureau C, Hanoun N, Torrisani J, Vinel JP, Buscail L, Cordelier P. Expression and Function of Kruppel Like-Factors (KLF) in Carcinogenesis. Curr Genomics. 2009, 10: 353-360.

51. Fu DZ, Cheng Y, He H, Liu HY, Liu YF. The fate of Krüppel-like factor 9-positive hepatic carcinoma cells may be determined by the programmed cell death protein 5. Int J Oncol. 2014, 44:153-160.

52. Sun J, Wang B, Liu Y et al. Transcription factor KLF9 suppresses the growth of hepatocellular carcinoma cells in vivo and positively regulates p53 expression. Cancer Lett. 2014, 355:25-33.

53. Dillon LM, Miller TW. The PREX1/Rac signaling axis: Potential as a biomarker and therapeutic target in breast cancer. Mol Cell Oncol. 2015 Apr 30,2(3):e996016. DOI: 10.1080/23723556.2014.996016.

54. Montero JC, Seoane S, Ocaña A, Pandiella A. P-Rex1 participates in Neuregulin-ErbB signal transduction and its expression correlates with patient outcome in breast cancer. Oncogene 2011, 30: 1059-1071.

55. Lindsay CR, Lawn $S$, Campbell $A D$ et al. P-Rex 1 is required for efficient melanoblast migration and melanoma metastasis. Nat. Commun. 2011, 2, 555. DOl:10.1038/ncomms1560.

56. Qin J, Xie Y, Wang B et al. Upregulation of PIP3-dependent Rac exchanger 1 (P-Rex1) promotes prostate cancer metastasis. Oncogene 2009, 28: 853-1863.

57. Xiao W, Zhang Q, Jiang F, Pins M, Kozlowski JM, Wang Z. Suppression of prostate tumor growth by U19, a novel testosterone-regulated apoptosis inducer. Cancer Res. 2003, 63:4698-4704.

58. Xiao W, Zhang Q, Habermacher $\mathrm{G}$ et al. U19/Eaf2 knockout causes lung adenocarcinoma, B-cell lymphoma, h[epatocellular carcinoma and prostatic intraepithelial neoplasia. Oncogene. 2008, 27:1536-1544.

59. Ai J, Pascal LE, Wei L et al. EAF2 regulates DNA repair through Ku70/Ku80 in the prostate. Oncogene. 2017, 36:2054-2065.

60. Liu JX, Zhang D, Xie X et al. Eaf1 and Eaf2 negatively regulate canonical Wnt/ $\beta$-catenin signaling. Development. 2013, 140:1067-1078. 
61. Su F, Pascal LE, Xiao W, Wang Z. Tumor suppressor U19/EAF2 regulates thrombospondin-1 expression via p53. Oncogene. 2010, 29:421-431.

\section{Figures}

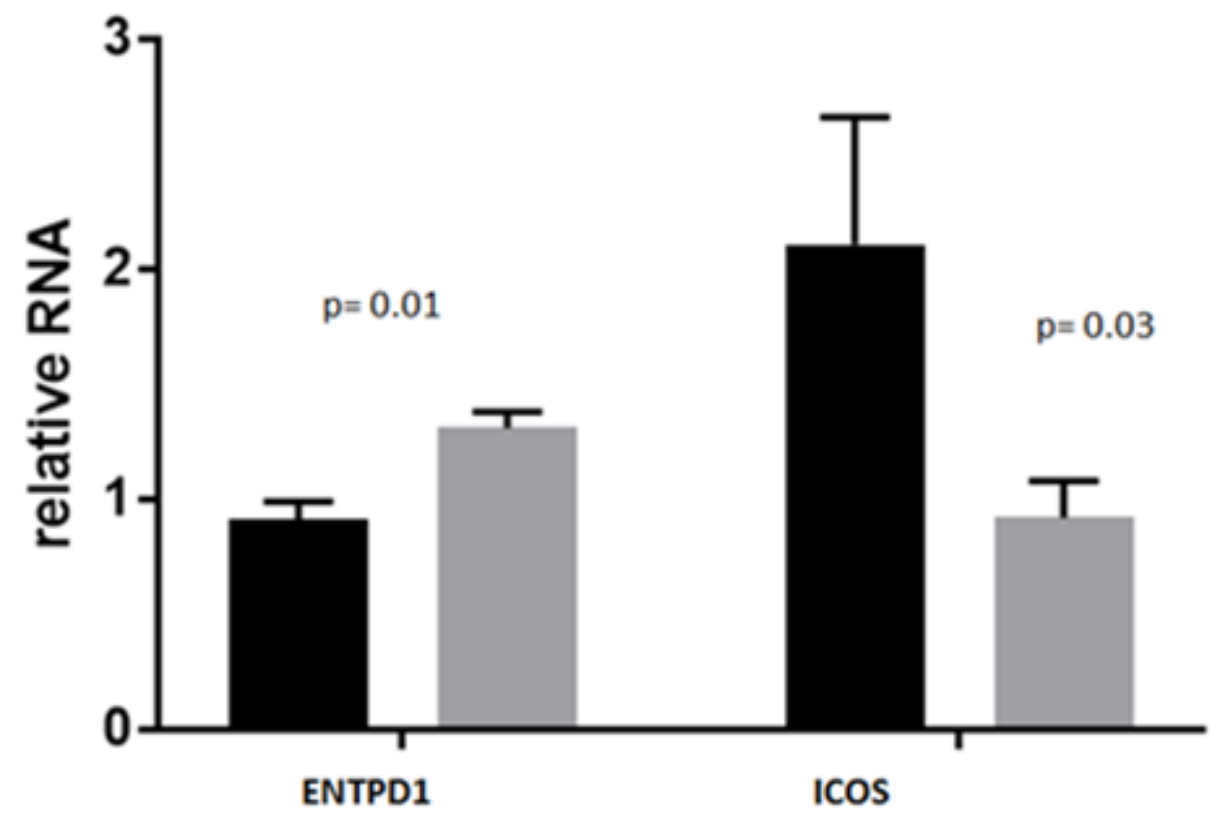

\section{Figure 1}

Results of the amplification with RT-qPCR of the genes resulted modified at the microarray assay, in the comparison at T0 of patients with CR (black) and PR (grey) (Mean+SEM of relative mRNA) 

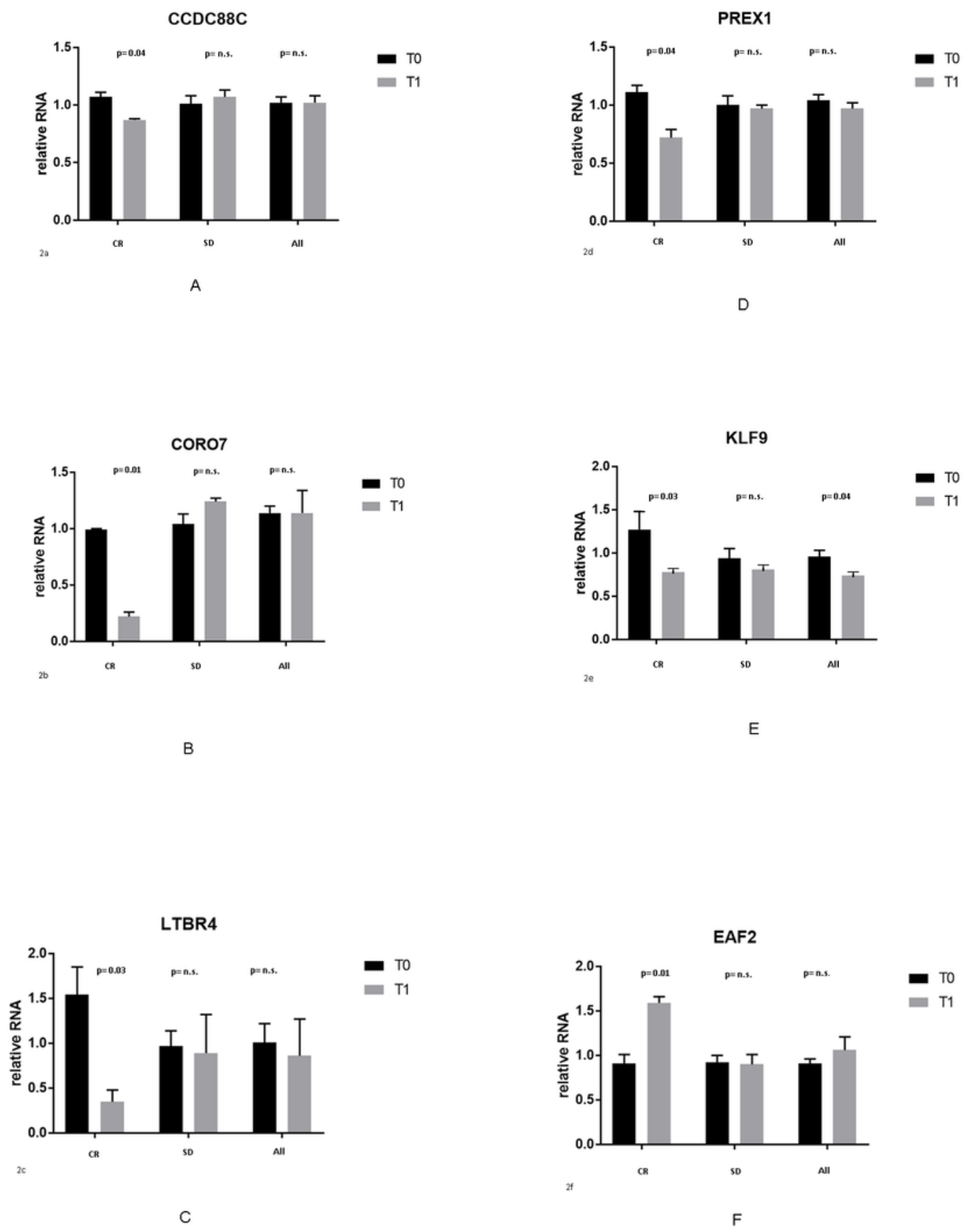

Figure 2

Results of the amplification with RT-qPCR of the genes resulted modified at the microarray assay, in the comparison between T0 and T1 on the entire population and in single groups of patients (Mean+SEM of relative mRNA) (a, CCDC88C; b, COR07; c, LTBR4, d, PREX1; e, KLF9; f, EAF2).

\section{Supplementary Files}


This is a list of supplementary files associated with this preprint. Click to download.

- SupplementaryTable1a.docx

- SupplementaryTable1B.docx

- SupplementaryTable1C.docx

- SupplementaryTable1D.docx 